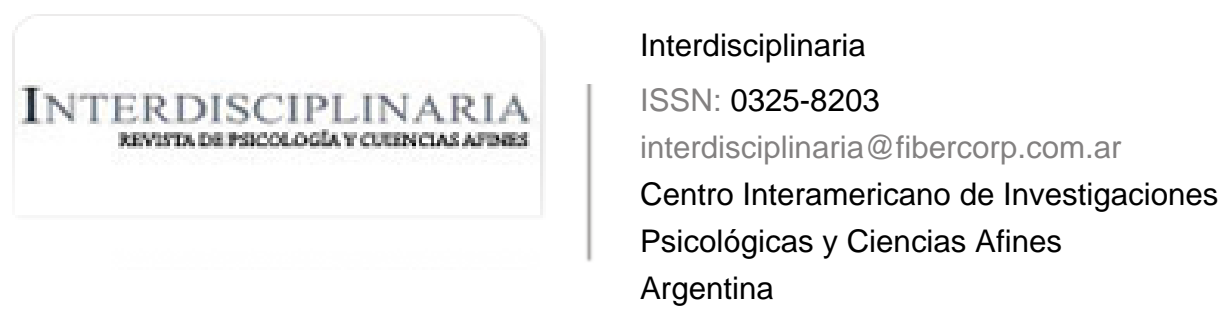

Ortiz Benavides, Fedra Lorena; Fernández Izquierdo, Pablo

Diseño instruccional para argumentación científica en línea

Interdisciplinaria, vol. 33, núm. 2, 2016, pp. 231-249

Centro Interamericano de Investigaciones Psicológicas y Ciencias Afines

Buenos Aires, Argentina

Disponible en: http://www.redalyc.org/articulo.oa?id=18049289003

- Cómo citar el artículo

Número completo

- Más información del artículo

- Página de la revista en redalyc.org

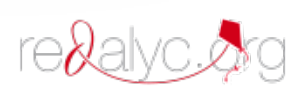

Sistema de Información Científica

Red de Revistas Científicas de América Latina, el Caribe, España y Portugal Proyecto académico sin fines de lucro, desarrollado bajo la iniciativa de acceso abierto 


\title{
DISEÑO INSTRUCCIONAL PARA ARGUMENTACIÓN CIENTÍFICA EN LÍNEA
}

\author{
INSTRUCTIONAL DESIGN FOR SCIENTIFIC ARGUMENTATION ON LINE
}

\author{
Fedra Lorena Ortiz Benavides* y Pablo Fernández Izquierdo**
}

\author{
*Licenciada en Biología, Magister en Didáctica de la Biología y Doctora en Educación, Tecnología Educativa y \\ Educación a Distancia. Docente Asistente Adscrita a la Escuela de Ciencias Básicas, Tecnología e Ingeniería de la \\ Universidad Nacional Abierta y a Distancia (UNAD) de Colombia. E-Mail: fedra.ortiz@unad.edu.co \\ Carrera 15. N. 28-45. Sector Bombona. Pasto. Colombia. \\ **Licenciado en Biología, Magister en Microbiología y Doctor en Microbiología. Docente Asociado Adscrito en la \\ Facultad de Ciencias Exactas y Naturales de la Universidad de Nariño. \\ Universidad Nacional Abierta y a Distancia (UNAD) y Universidad de Nariño. \\ Nariño - Colombia
}

\section{REsumen}

El propósito de la investigación realizada fue evaluar la efectividad de la enseñanza de procesos estructurados de argumentación en el desarrollo de la argumentación científica de estudiantes inscritos en un curso virtual de Biotecnología.

Con un diseño experimental se compararon dos grupos de estudiantes, solo uno recibió orientación sobre procesos de argumentación. Ambos grupos participaron en un foro de trabajo colaborativo en línea.

En ambos grupos la evaluación de la competencia argumentativa se realizó aplicando el instrumento de evaluación para argumentación dialógica en línea, propuesta por Clark y Sampson (2008); este modelo consta de tres categorías: estructura argumentativa, calidad de la argumentación y nivel de oposición al que llega el grupo.

Para realizar el experimento se construyó un diseño instruccional basado en la argumentación dialógica. El primer componente del diseño corresponde a la enseñanza del modelo argumentativo. El segundo elemento es el contexto de aprendizaje a partir del cual se desarrolla la interacción y el tercero corresponde a las mediaciones tecnológicas. El diseño instruccional se incorporó en el curso virtual de Biotecnología alojado en la plataforma tecnológica de la Universidad, la cual utiliza Moddle 2X.

Los resultados indicaron que el $60 \%$ de los estudiantes del grupo experimental utilizó todas las categorías argumentativas de Toulmin; al contrario, ningún estudiante del grupo control obtuvo un nivel de estructuración con todas las categorías. Asimismo, la enseñanza explícita de procesos estructurados de argumentación provoca mejor desempeño en el foro de trabajo colaborativo en aspectos como nivel de calidad de los argumentos y nivel de oposición.

Palabras clave: Argumentación científica; Competencia argumentativa; Modelo argumentativo de Toulmin; Argumentación dialógica; Foros de discusión en línea; Enseñanza de las ciencias.

\section{ABSTRACT}

Teaching argumentation is a strategy of great interest within the community involve in the study of didactic in science since there are numerous studies showing the relationship between the development of scientific thought and the argumen- 
tation structured processes (Khun, 2008), however in virtual learning environments teaching argumentation is a relatively new issue and therefore constitutes a challenge for the instructional designer of the virtual course. Collaborative learning in virtual environments is a strategy implemented in recent years in such contexts under the assumption that computer-mediated and asynchronously communication facilitates the maintenance of dialogue (Schwarz \& Glassner, 2003). However, studies indicate that these students' dialogues in virtual forums can be weak and based on beliefs not justified instead of using reasons articulated between theory and evidence, otherwise can be characterized because students within the group accept explanations without paying enough attention on the relationship between a statement and the available evidence and therefore readily accepted arguments without making opposition. This condition can be explained because in the context of collaborative internet the problems increase due to issues like complexity of interfaces, decreasing communication channels regarding classroom situations, or the ease of students to mask his inactivity within collaborative groups (Peñaloza, García \& Espinoza, 2011).

Although most researchers agree that the dialogic argumentation promotes the acquisition of scientific concepts, also they say it is difficult to integrate the teaching of argumentation structured processes into a virtual course of experimental science as well as evaluating appropriation of concepts through arguments.

In this paper the results of an investigation conducted to evaluate the effectiveness of an instructional design based on structured argument process in the development of the argumentative capacity of higher education students enrolled in an online course biotechnology is presented.

The po pulation under investigation corresponds to higher education students of both sexes who are enrolled in a Biotechnology online course offered by a distance university in Colombia

Instructional design incorporated three elements: the first is the teaching of Toulmin argumentative model, the second element incorporated was the situated learning context from which the interaction is developed and for this study corresponds to a Biotechnology theme and the third component included the design and development of techno-didactic mediations to promote educational interactivity where virtual learning objects, guide activities and assessment rubrics were included. These elements were incorporated into the virtual course within the Moodle 2.1 platform. Moreover, learning strategy based on dialogic argument was developed in four stages: (a) scientific research, (b) argumentative paper development, (c) counter argument, and (d) scientific consensus.

To evaluate the effectiveness of instructional design and online learning strategy based on dialogic argumentation an experimental design was conducted with a control group and post-test. The assessment of the argumentative capacity was performed by applying the online assessment tool for dialogic argumentation proposed by Clark and Sampson (2008). This model evaluates the argumentative process in the virtual forum both individually and group. Data were analyzed with nonparametric statistical tests.

The research results indicated significant differences in the level of argumentative competence in the experimental group compared with the control group. The experimental group had higher ratings on their ability to structure texts, support arguments within the group and make reasoned opposition to reach consensus. Also, results of this research support the design and learning strategy based on the argument for virtual environments and shown it can be effective to increase the academic performance of students participating in collaboration online forums.

Students in the control group did not performed opposition, and, most students in the experimental group achieved level three opposition on the scale of Clark and Sampson (2008), this aspect indicates the need to develop strategies to increase the level of opposition.

Key words: Scientific argumentation; Argumentative skill; Toulmin's Argument Pattern; Dialogic argument; Discussion forums online; Science education.

\section{INTRODUCCIÓN}

El estudio de la capacidad argumentativa asociada al desarrollo del pensamiento cien- 
tífico es un tema que recobró auge a partir de la última década del siglo pasado. Según Duschl, Ellenbogen y Erduran (1999) este fenómeno se debe a posturas epistemológicas que reconocen la ciencia como una cultura en permanente transformación, cuyo carácter evolutivo implica entender que la racionalidad se encuentra ligada a la flexibilidad intelectual o disponibilidad al cambio (Toulmin, 1958).

El estudio de la argumentación se remonta casi a los orígenes de la Filosofía misma a través de la retórica, la dialéctica y la lógica; no obstante, el impulso del estudio de la teoría de la argumentación se evidencia desde la última década del Siglo XX influenciada por la pragmática expuesta en las publicaciones realizadas por Perelman y Toulmin, quienes formularon modelos y reglas para la argumentación (Marafioti \& Santibáñez, 2008). Desde esta perspectiva, la argumentación consiste en un conjunto de estrategias discursivas que tienen por objetivo demostrar la validez de una afirmación.

Para Toulmin (1958) los argumentos son mecanismos justificatorios, no mecanismos persuasivos o deliberativos como hasta ese momento lo consideraba la lógica formal, en la cual un argumento era válido si producía los silogismos correctos. En cambio, desde la pragmática los argumentos pueden diferir enormemente según el campo al que pertenezcan y el tipo de evidencia que justifican el argumento. Por ejemplo, la predicción meteorológica recurre a un tipo de argumento y evidencias muy diferentes para elaborar un juicio moral (Merafioti \& Santibáñez, 2008).

En el modelo de Toulmin (conocido como Toulmin's Argument Pattern - TAP) se identifican elementos invariables que se encuentran en la mayoría de los argumentos independientemente del contexto en el que se sitúan, tales como: (1) datos, (2) conclusión, (3) garantía, (4) justificación o respaldo, (5) calificador modal y (6) refutación. También hay una serie de elementos variables que cambian en función del área de conocimiento. En este modelo la validez de un argumento es sustentada, necesariamente por la coherencia de su justificación. Este esquema se utiliza en la didáctica de las ciencias para evaluar la cali- dad de los argumentos construidos por los estudiantes (Simon, Erduran \& Osborne, 2006).

Igualmente desde la Psicología Cognitiva, Kuhn (2008) considera la argumentación como un proceso cognitivo donde la persona construye sus propias teorías y evalúa las de otros; en los dos casos implica utilizar evidencias y relacionarlas con la teoría para sustentar sus afirmaciones. De esta manera se establece una distinción entre argumento como producto y argumentación como proceso cognitivo susceptible de enseñarse en el ámbito educativo.

Además en la enseñanza de las Ciencias, Driver, Newton y Osborne (2000) reconocen la argumentación como una actividad dialógica o de voz múltiple que tiene lugar cuando el individuo en el seno de un grupo examina diferentes perspectivas para llegar a acuerdos sobre las afirmaciones. Por lo tanto, la argumentación se define como un proceso complejo de la construcción de argumentos (Osborne, 2004), ya sea individualmente o dentro de un grupo, que se puede expresar verbalmente o por escrito (Driver et al., 2000).

Por lo tanto, en el ámbito educativo además de propiciar espacios de discusión, es necesario enseñar a los estudiantes a comprender los fenómenos científicos a partir del análisis de las evidencias (Jiménez-Aleixandre, 2005), como también aplicar procesos estructurados de argumentación para formular tesis y analizar los planteamientos de otros En este sentido, Sánchez, A., Sánchez, L., Méndez y Puerta (2013) reconocen la importancia de la enseñanza de la argumentación en las aulas de educación superior, para comprender los elementos que permitan asumir el texto como una actividad no solamente de comunicación, sino cognitiva que conlleva un acto comunicativo intencional y que puede brindar al docente una variedad de elementos y saberes para evaluar un texto con parámetros claros y suficientes.

Sin embargo, la implementación de estrategias para la enseñanza de la argumentación en la educación virtual es un proceso diferente a la educación presencial porque en contextos de colaboración en internet los problemas 
de comunicación aumentan debido a la complejidad de las interfaces, el tiempo de respuesta que otorga un carácter asincrónico a la interacción o la facilidad de los estudiantes para enmascarar su inactividad en los grupos colaborativos (Peñaloza, García \& Espinoza, 2011).

Por otra parte, Andriessen, Baker y Suthers (2003) examinaron en un ambiente web los tipos de argumentos que apoyan el aprendizaje colaborativo. Según este estudio, los argumentos tienen dos tipos de estructura: la primera corresponde a la estructura de los argumentos individuales que incluye: (1) los datos que conforman la evidencia en la cual el argumento se basa, (2) una declaración que afirma una posición y (3) una razón que indica por qué los datos apoyan las declaraciones. Esta codificación coincide con las categorías establecidas en el Modelo Argumentativo propuesto por Toulmin, Rieke y Janik (1984). La segunda estructura corresponde a las secuencias de la argumentación dentro del grupo e involucra: (1) argumentos, (2) argumentos en contra y (3) argumentos de integración (Litao, 2000).

En consecuencia, investigadores como Kollar, Fischer y Hesse (2010) proponen que para la enseñanza de las ciencias en ambientes virtuales basados en la argumentación, se debe declarar explícitamente la codificación de los mensajes de tal manera que facilite el seguimiento, la evaluación y la construcción de un debate argumentativo en línea. El estudio realizado por Clark y Sampson (2008) en un ambiente virtual diseñado bajo las anteriores condiciones demostró que los preconceptos de los estudiantes entran en conflicto con las secuencias que se sistematizan en el ordenador. En otras palabras, significa que mientras algunos participantes saben cómo realizar una sustentación para exponer sus razones de forma explícita en los argumentos y lo que deben hacer para realizar una buena argumentación, contra-argumentación o utilizar argumentos de integración, otros no tienen ese conocimiento y desarrollan argumentos que se basan en alegaciones subjetivas o simplemente se abstienen de participar en la discusión.
Con respecto al diseño instruccional para enseñar la argumentación dialógica en ciencias, Lin, Shear, Bell y Slotta (1999) realizaron un estudio donde demuestran que el diseño de entornos virtuales que apoyan la participación de la argumentación dialógica, es efectivo no solamente para el aprendizaje de la argumentación científica, sino también para el aprendizaje de los conocimientos científicos mediante la participación activa en esa argumentación. Sin embargo, Shwarz y Perret (2006) sostienen que es difícil diseñar actividades en línea que combinen la argumentación dialógica con la investigación, dados los problemas de comunicación que se pueden presentar en un ambiente asincrónico.

En este sentido, Kollar y colaboradores (2010) afirman que los diseñadores deben tener en cuenta muchos factores en el momento de desarrollar actividades que involucren la argumentación dialógica, independientemente del modelo de argumentación que se aplique, tales como (a) la orientación para la participación, (b) la dinámica y la secuencia de las intervenciones y (c) la evaluación tanto de los aportes individuales como de las discusiones que desarrolla el grupo.

Paralelamente a las propuestas metodológicas para la implementación de los debates en línea, también se proponen modelos para evaluar la dinámica de comunicación dentro de un foro virtual desde diversas perspectivas, tales como los modelos propuestos por Guanawardena, Lowe y Anderson (1997) y Mercer (2001), entre otros. Clark y Sampson (2008) plantean un modelo de análisis de la calidad de los argumentos inspirado en el modelo de Toulmin (1958) y Erduran y colaboradores (2006), además incorporan y aumentan los esquemas de codificación desarrollados por varios investigadores para analizar la estructura de la argumentación de los estudiantes de ciencias (Jiménez, Bugallo $\&$ Duschl, 2000; Osborne, 2004; Simon et al., 2006). Igualmente este modelo es utilizado como instrumento de evaluación en diferentes investigaciones, como la realizada por Skoumios (2008) que aplicó el instrumento para estudiar el efecto del conflicto sociocognitivo en el desarrollo de la capacidad ar- 
gumentativa en estudiantes de Física. Igualmente, Kinsley y Firuz (2009) y Jeong (2011) utilizaron este modelo para estudiar el aprendizaje cognitivo en grupos colaborativos en ciencias.

Las investigaciones destinadas a estudiar las habilidades de argumentación de los escolares sugieren que la mayoría de ellos enfrenta dificultades relacionadas con su capacidad argumentativa tanto individualmente como durante la interacción dialógica, aún después de su exposición a ambientes de aprendizaje que tienen como objetivo promover las habilidades de argumentación como es el caso de foros de interacción colaborativa en línea.

Por lo tanto, el propósito de la investigación realizada estuvo centrado en diseñar un entorno de aprendizaje basado en la argumentación dialógica y situado en un curso virtual de Biotecnología, con el que se pretende responder la siguiente pregunta: ¿En qué medida la interacción de los estudiantes en un ambiente colaborativo y dialógico especialmente diseñado para el aprendizaje en línea mejora la promoción de las competencias de argumentación?

\section{Metodología}

\section{PARTICIPANTES}

Participaron 60 estudiantes de la Carrera de Ingeniería de Alimentos, matriculados en un curso virtual de Biotecnología de la universidad donde se realizó el estudio, sin atender el género o ubicación geográfica. Teniendo en cuenta que el promedio de matrícula del curso era de 95 estudiantes, se tomó para el estudio dicha muestra representativa de 60 alumnos para un nivel de confiabilidad del 95\%.

La conformación de los grupos se realizó a partir de un muestreo aleatorio simple dado que las unidades maestrales son homogéneas con respecto a la característica poblacional a estudiar. Se distribuyeron en dos grupos de 30 estudiantes cada uno, grupo experimental (A) y grupo control (B). La distribución la realizó la oficina de registro y control de la universidad, por lo tanto ni los docentes ni los estudiantes incidieron en la conformación de los mismos.

\section{INSTRUMENTOS}

El modelo de Clark y Sampson (2008) se utilizó para estudiar la competencia argumentativa constituida por tres categorías. La primera corresponde a la estructura argumental que realizan los estudiantes individualmente y consistió en el seguimiento de los comentarios individuales de acuerdo con el uso de las categorías de argumentación propuestas en el Modelo de Toulmin (1958) como se indica a continuación:

Datos: Hechos o informaciones factuales que se invocan para justificar y validar la afirmación.

Aserción: Es la hipótesis que se va a defender, el asunto a debatir, a demostrar o a sostener en forma oral o escrita. Expresa la conclusión a la que se quiere arribar con la argumentación, el punto de vista que la persona quiere mantener, la proposición que se aspira que otro acepte. Indica la posición sobre determinado asunto o materia.

Garantía: Son razones (reglas, principios) que se proponen para justificar las conexiones entre los datos y la conclusión. Su función es de conexión entre la evidencia y la aserción.

Respaldo: Es el conocimiento básico que permite asegurar la justificación.

Cualificador modal: Aporta un comentario implícito de la justificación; de hecho, es la fuerza que la justificación confiere a la argumentación.

Reserva: Señala las circunstancias en que las justificaciones no son ciertas.

La segunda categoría corresponde a la evaluación de la calidad argumentativa de acuerdo al modelo propuesto por Clark y Sampson (2008) y realizada por el grupo de trabajo colaborativo. Está conformada por cuatro niveles que son los siguientes:

Nivel 0: En la discusión no se incluyen intentos de justificar la posición ante el grupo.

Nivel 1: Los estudiantes sencillamente repiten o reformulan los fundamentos utilizados por otros estudiantes sin añadir algo nuevo. 
Nivel 2: Los estudiantes refieren por lo menos una fuente de información, como un dato o evidencia confiable a partir de una experiencia personal, una actividad de laboratorio, datos empíricos, referencian autores o libros o dan un ejemplo.

Nivel 3: El grupo referencia y apoya sus argumentos con múltiples fuentes, analiza los datos empíricos y los relaciona con la teoría.

La tercera categoría mide el grado general de la competencia argumentativa que logra el grupo mediante una jerarquía basada en la oposición, con cinco niveles de acuerdo al modelo propuesto por Clark y Sampson (2008) y son los siguientes:

Nivel 0: No hay oposición.

Nivel 1: Las oposiciones son simples y no están fundamentadas con motivos o refutaciones.

Nivel 2: Las argumentaciones implican demandas o reconvenciones motivadas pero no hay réplicas.

Nivel 3: Presenta demandas o reclamaciones, pero una sola refutación desafía la tesis.

Nivel 4: Múltiples refutaciones que cuestionan la tesis, pero no incluyen fundamentos o evidencias para apoyar el reclamo.

Nivel 5: Presenta múltiples refutaciones respaldadas con fundamentos que debilita la tesis.

\section{Procedimiento}

Este estudio se desarrolló en dos etapas: la primera correspondió al diseño de una actividad de aprendizaje colaborativo basado en la argumentación dialógica para el curso virtual de Biotecnología y la segunda consistió en el diseño y ejecución del experimento y evaluación de la competencia argumentativa.

\section{Diseño INSTRUCCIONAL EN EL AULA VIRTUAL}

Se dispuso un espacio en el curso virtual de Biotecnología alojado en la plataforma tecnológica de la universidad, la cual utiliza Moddle 2X. Esta plataforma cuenta con diferentes herramientas que facilitan la interac- ción didáctica durante el proceso de aprendizaje, entre ellas el foro académico. El diseño instruccional se especificó para cada grupo como se presenta en la Figura 1.

\section{CONSTRUCCIÓN DEL DISEÑO INSTRUCCIONAL BA- SADO EN LA ARGUMENTACIÓN DIALÓGICA}

En este diseño se conjugan tres elementos que se relacionan entre sí para garantizar la comunicabilidad, la interacción, la interactividad didáctica y la significancia del aprendizaje, cuyas relaciones se observan en la Figura 2.

El primer componente del diseño instruccional corresponde a la enseñanza del modelo argumentativo, el cual se constituye en la guía para la codificación y decodificación en el proceso argumentativo.

El segundo elemento considerado en el diseño es el contexto de aprendizaje a partir del cual se desarrolla la interacción, donde se tuvieron en cuenta los preconceptos, los conocimientos específicos situados en la Biotecnología y la estrategia didáctica para garantizar la significatividad del aprendizaje. En concordancia con lo anterior, se les presentó a los estudiantes un problema real de interés común en su formación profesional con el fin de propiciar la interacción.

El tercer elemento que se incorporó al diseño son las mediaciones tecnológicas, puesto que en un ambiente virtual los medios son los instrumentos físicos, semióticos o signos por medio de los cuales se facilitan o se hacen más eficientes las formas de acción para el aprendizaje. Por lo tanto, todos los recursos se diseñaron con una intencionalidad didáctica.

\section{IMPLEMENTACIÓN DEL DISEÑO INSTRUCCIONAL EN EL AULA VIRTUAL}

Se realizó en dos momentos.

Momento 1: Instrucción previa en argumentación científica.

Para ello se utilizaron diversos recursos: (a) información sobre la argumentación científica, (b) explicación del Modelo Argumentati- 
vo de Toulmin - MAT, (c) ejemplo de la forma como se debe estructurar un texto argumentativo basado en el MAT y (d) ejercicio de análisis de un artículo científico identificando cada una de las categorías del MAT. Esta intervención tubo carácter individual y asincrónico, por lo tanto la actividad estuvo abierta en el aula virtual durante una semana para que los estudiantes entregaran el producto final.

Momento 2: Estrategia didáctica basada en argumentación dialógica.

La estrategia de aprendizaje basada en la argumentación dialógica se fundamenta en reconocer que la argumentación es un proceso cognitivo que puede ser enseñable, pero igualmente se reconoce que en ciencias, el proceso de argumentación es necesario para construir nuevo conocimiento a partir de la evaluación de la evidencia y de la rigurosidad con que se construyen y aceptan los argumentos; es así que la estrategia se estructuró en cuatro fases como se explica a continuación y se muestra en la Figura 3.

\section{1.- Etapa de fundamentación científica: Esta} etapa tiene el propósito de que los estudiantes antes de ingresar a una discusión tengan la suficiente claridad conceptual para participar en el foro asertivamente. Se orienta la actividad a partir de preguntas sobre el tema específico con diferentes niveles de complejidad, que van desde la definición de conceptos al análisis y a la aplicación de los mismos. Para ello, los estudiantes contaron con los siguientes recursos: (a) objetos virtuales de aprendizaje sobre enzimas de interés alimentario y procesos de fermentación, (b) vínculos interactivos a diferentes artículos científicos sobre el tema de estudio y (c) multimedia demostrativa de un laboratorio virtual. Al ingresar a esta multimedia interactiva los estudiantes pueden observar los procedimientos de laboratorio y obtener resultados sobre el problema que deben solucionar. Entregaron el producto por medio del recurso Tarea.

2.- Etapa de elaboración de la ponencia científica: Se presentó a los estudiantes un objeto virtual de aprendizaje donde se con- textualiza la situación problema de un experimento relacionado con la evaluación de los componentes de un medio de cultivo para la producción de la enzima alfa amilasa por medio de un microorganismo. En el problema se muestran tres posibles hipótesis que pueden explicar el fenómeno de estudio. El ejercicio consistía en que los estudiantes individualmente y teniendo en cuenta la teoría y los datos del experimento, debían escoger una de las hipótesis planteadas y a partir de ésta desarrollar una ponencia científica. Para realizar esta actividad se les entregó una Guía de Actividades la cual indicaba explícitamente los pasos a seguir para realizar la ponencia individual de acuerdo con el Modelo Argumentativo de Toulmin. La entrega del producto lo socializó en el foro académico.

\section{3.- Etapa de evaluación de los argumentos y} contra argumentación: Para esta etapa se crearon dentro de la plataforma, foros de trabajo colaborativo para que cada estudiante evaluara tanto la estructura argumental como la evidencia teórica y empírica que respaldó la hipótesis de la ponencia enviada por uno de sus compañeros. A su vez, debían construir una contra-argumentación debidamente fundamentada de la hipótesis formulada.

4.- Construcción del consenso cientifico: En esta etapa se busca que se lleve a cabo la argumentación dialógica dentro del grupo colaborativo, de tal manera que en el proceso de argumentación y contra-argumentación los estudiantes articulen la solución del problema a partir de premisas que admitan consenso, es decir, que puedan ser comprendidas, evaluadas y que se correspondan con la solución del problema expuesto. Esta actividad fue realizada en el foro de trabajo colaborativo. Para ello, se les entregó una guía orientativa para que evaluaran tanto las ponencias como las contra-argumentaciones realizadas en la etapa anterior y que a partir de esta evaluación llegaran a establecer un acuerdo sobre la hipótesis que mejor podría explicar el experimento. Igualmente, se les entregó una guía que indicaba cómo debían interactuar con sus compañeros para llegar al consenso. El texto 
final fue consolidado y enviado al foro de trabajo colaborativo.

\section{DISEÑO DEL EXPERIMENTO}

La segunda etapa se desarrolló a partir de la aplicación de un experimento con el grupo control y solamente postest (Campbell \& Stanley, 1995) con el fin de evaluar la efectividad del diseño instruccional en el incremento de la capacidad argumentativa en un ambiente virtual de aprendizaje. Para ello, los participantes se dividieron en dos grupos A y B de 30 estudiantes cada uno. Para facilitar el seguimiento de las intervenciones, estos a su vez se dividieron en seis subgrupos de cinco estudiantes cada uno (A1, A2, A3, A4, A5 у A6; B1, B2, B3, B4, B5 у B6).

El grupo experimental A desarrolló la actividad de acuerdo al diseño instruccional basado en la argumentación dialógica, mientras que el grupo control B realizó la actividad como tradicionalmente se desarrolla en los foros de trabajo colaborativo en el aula virtual de Biotecnología.

\section{EVALUACIÓN DE LA COMPETENCIA ARGUMENTATIVA}

Para el seguimiento de las categorías argumentales en la ponencia individual, se codificaron las intervenciones como ausencia y presencia en cada una de las categorías argumentales presentadas en los textos de los estudiantes de ambos grupos. Con el fin de cuantificar el número de estudiantes por grupo que utilizaron en sus argumentos todas las categorías argumentales de Toulmin, se realizó un estudio comparativo de las frecuencias por análisis exploratorio de la distribución de esas frecuencias por grupo y entre grupos.

Para el análisis de los datos de las categorías Calidad conceptual de los comentarios y Nivel de oposición que se presenta en el grupo, se realizó una categorización de las intervenciones que los estudiantes efectuaron en cada grupo y una vez clasificadas por niveles dichas intervenciones, se realizó una prueba de chi cuadrado para determinar la variabili- dad dentro del grupo. Por último entre los grupos experimental y control se aplicó una prueba estadística de significancia KolmogorovSmirnov para muestras independientes, dado que se trata de variables de tipo ordinal.

\section{REsultados}

PRESENCIA DE LAS CATEGORÍAS ARGUMENTALES DEL MAT EN LOS TEXTOS ENVIADOS POR LOS ESTUDIANTES

El 60\% de los estudiantes del grupo experimental utilizó todas las categorías argumentativas de Toulmin en sus intervenciones; al contrario, ningún estudiante del grupo control obtuvo un nivel de estructuración con todas las categorías. Por otra parte, al menos el $10 \%$ de los estudiantes del grupo experimental utilizó hasta tres categorías, aspecto no evidenciado en el grupo control donde el $53 \%$ de los estudiantes solamente utilizó hasta dos categorías.

Es de resaltar que entre los dos grupos no se presentaron diferencias significativas en la categoría datos; sin embargo, las categorías garantía, respaldo y reserva fueron superiores al $80 \%$ en el grupo experimental, mientras que en el grupo control, la categoría garantía no superó el $50 \%$ de presencia y las categorías respaldo y reserva se encontraron en un porcentaje inferior al 30\%.

La categoría cualificador modal registró menor valor de presencia en los textos argumentativos, con un porcentaje de presencia correspondiente al $66.7 \%$ en el grupo experimental; no obstante, en el grupo control esta categoría solo alcanzó un $10 \%$ de presencia. En la prueba $Z$ de comparación de proporciones se encontró que en presencia de las categorías argumentativas garantía, respaldo, reserva y cualificador modal se presentaron diferencias significativas entre los dos grupos, a un nivel de significancia de .05 .

\section{RESULTADOS DE LA CALIDAD CONCEPTUAL DE LAS IN- TERVENCIONES}

En el Gráfico 1 se puede observar la clasificación del nivel conceptual alcanzado por 
ambos grupos. Muestra que el 63.3\% de estudiantes del grupo experimental (A) se ubicó en el Nivel 3, mientras que el $60 \%$ de los estudiantes del grupo control (B) se ubicó en el Nivel 1. Igualmente, se puede observar que ningún sujeto del grupo A se ubicó en el Nivel 0 , mientras que el $6.7 \%$ del grupo B se clasificó en este nivel. Además, el gráfico muestra que solamente 4 estudiantes del grupo $\mathrm{B}$, correspondiente al $13.3 \%$, se ubicaron en el máximo nivel.

A través de la prueba chi cuadrado de bondad de ajuste se encontró que no existen diferencias significativas entre estudiantes de un mismo grupo $(p<.05)$. En consecuencia se aplicó el estadístico de Kolmogorov-Smirnov $(Z=2.195)$ obteniendo una $p=.000$, por lo tanto el nivel conceptual difiere significativamente entre los dos grupos a un nivel de significancia del 95\%.

\section{Resultados DEL NIVEL DE OPOSICIÓN ALCANZADO POR LOS GRUPOS}

En el Gráfico 2 se indica la ubicación del nivel de oposición alcanzado por los estudiantes de ambos grupos. Los estudiantes del grupo experimental se ubicaron con mayor frecuencia en el Nivel de oposición 2 y 3 con un $36.7 \%$, respectivamente, seguidos del Nivel 4 con un $16.7 \%$, y el nivel 1 con un $3.3 \%$, en tanto ninguno se ubicó en el Nivel 5. Los estudiantes del grupo control se ubicaron con mayor frecuencia en el Nivel 0 con un $43.3 \%$, seguidos del Nivel 1 con un $33.3 \%$, el Nivel 2 con un $16.7 \%$, el Nivel 3 con un $6.7 \%$ y ninguno en el Nivel 4.

Con el fin de comparar la distribución de la variable Nivel de oposición en ambos grupos se realizó una prueba de bondad de ajuste para ambos grupos. Su resultado es para el Grupo A: $\chi^{2}=15.333, g l=4$, siendo su nivel crítico igual a .004 y dado que es menor que .05 , se puede rechazar la hipótesis de bondad de ajuste; para el Grupo B: $\chi^{2}=9.733, g l=3$ y su nivel crítico, .021, como es menor que .05 , se puede afirmar que la variable Nivel de oposición no presenta una distribución normal para ambos grupos.
El valor del estadístico de KolmogorovSmirnov es igual a 2.582 y su nivel crítico bilateral, .000. Puesto que este valor es menor que .05 , se concluye que la variable Nivel de oposición difiere significativamente en el grupo experimental y en el grupo control, a un nivel de significancia del $95 \%$.

\section{Discusıón}

Los resultados obtenidos del seguimiento a las intervenciones individuales realizadas durante el foro virtual, indican que la mayoría de los estudiantes del grupo experimental, a diferencia de los estudiantes del grupo control, identificaron las funciones que cumple cada categoría del MAT dentro de un texto argumentativo y las utilizaron para realizar sus intervenciones. Así queda demostrado que es necesario enseñar explícitamente procesos de argumentación para que los estudiantes desarrollen textos estructurados y defiendan sus afirmaciones. No basta con proponer debates en línea, puesto que en la mayoría de las ocasiones los estudiantes tienen dificultades para utilizar los datos y relacionar adecuadamente la evidencia con la teoría para explicar un fenómeno en ciencias.

Los resultados obtenidos están acordes con las investigaciones realizadas por Dionne, Taasoobshirazi, Hendricks y Hickey (2012), quienes encontraron que los estudiantes que fueron vinculados a una actividad de argumentación en línea utilizando el MAT, alcanzaron mejor grado de estructuración de sus textos argumentativos que los del grupo control. Estos hallazgos también coinciden con los estudios de Wen, Wei y Shing (2012), quienes observaron que estudiantes que participaron en una actividad argumentativa en línea, en la cual recibieron orientación para organizar sus intervenciones, lograron mejores resultados de aprendizaje que sus contrapartes del grupo control en cuanto al nivel del discurso en el grupo, el aprendizaje en grupo e individual.

Los resultados anteriores se pueden explicar porque según Jiménez y colaboradores (2000), el modelo argumentativo de Toulmin 
constituye una herramienta muy potente para estructurar el pensamiento científico, puesto que lo hace a partir del uso de la lógica formal. Además, Henao y Stipcich (2008) consideran que el MAT se encuentra concebido desde la epistemología evolutiva, lo que contribuye a que en clases de ciencias, se piense y se hable como científico. El hecho de que los estudiantes del grupo experimental (A) hayan utilizado las categorías argumentales de acuerdo al MAT y que en consecuencia sus argumentaciones se encuentren mejor estructuradas, contribuye a reafirmar los estudios anteriores.

Igualmente, que los estudiantes hayan construido un texto con las categorías argumentales supone un mayor esfuerzo cognitivo, puesto que necesariamente debían interrelacionar las diferentes categorías para acercarse a una hipótesis que explique de forma adecuada el fenómeno estudiado. Esto necesariamente los conduce a realizar diversas operaciones mentales como el análisis, la evaluación y la contratación de la evidencias que les permitió establecer relaciones entre los datos experimentales con la teoría científica que respalda la hipótesis escogida.

En cuanto a la evaluación de la calidad de los argumentos, los resultados muestran que los estudiantes del grupo experimental se ubicaron en un mayor nivel de conceptualización de la argumentación porque utilizaron los datos del experimento, los compararon con otros datos extraídos de los artículos científicos y además los relacionaron con la teoría y así justificaron su hipótesis. Sin embargo, la mayoría de los estudiantes del grupo control basaron su explicación únicamente en la teoría sobre los factores que pueden alterar la velocidad enzimática tales como la temperatura, el $\mathrm{pH}$, entre otros, sin tener en cuenta el diseño del experimento ni los datos del mismo. Igualmente, la discusión fue muy limitada y las intervenciones se restringieron a apoyar la posición de los compañeros sin añadir nada nuevo, incluso se evidenció una falta de preocupación por leer los artículos científicos que tenían para desarrollar el ejercicio.

Lo expuesto demuestra que los estudiantes, incluso de niveles de educación superior, presentan dificultades para interpretar resul- tados y relacionarlos con la teoría científica, lo que evidencia la necesidad de incorporar ejercicios explícitos en los cursos de ciencias que los convoque a evaluar la evidencia científica y puedan contrastarla con la teoría. Este hallazgo se encuentra en concordancia con los estudios realizados por Hogan, Nastasi y Pressley (1999) y Clark y Sampson (2008), quienes encontraron que los estudiantes que tienen instrucciones previas sobre argumentación, demuestran mayor claridad conceptual no sólo en los conceptos específicos sino también en los procesos argumentativos. Es decir que relacionan más claramente los datos con la teoría, son capaces de justificar su argumento y llegar a conclusiones coherentes.

Igualmente, Bouyias y Starvros (2012) demostraron que la enseñanza explícita de la argumentación y guiones de participación provocan efectos positivos en la capacidad argumentativa de los estudiantes que participan en un debate en línea. Por su parte, Belland (2010) demostró que el reto cognitivo que significa que los estudiantes resuelvan problemas en línea basados en la evidencia, les proporciona andamios conceptuales que permiten organizar mejor la tarea y estructurar las explicaciones.

El hecho de que los estudiantes del grupo experimental hayan alcanzado niveles más altos de conceptualización argumental, puede explicarse porque según Dole y Sinatra (1998) la enseñanza de la argumentación permite un procesamiento cognitivo más profundo, porque ellos deben considerar los argumentos y los contra-argumentos que realizan sus compañeros para llegar a una conclusión, lo que requiere un mayor esfuerzo cognitivo. Por lo tanto, estos autores sostienen que aunque los estudiantes pueden llegar a generar argumentos no tan críticos, incluso una intervención corta sobre procesos argumentativos, puede alterar significativamente su procesamiento cognitivo.

Un aporte importante de la estrategia aplicada en la cual se consideró una etapa de fundamentación científica, es que se demostró que cuando los estudiantes conocen previamente el tema y se les da la oportunidad de re- 
flexionar y contrastar los datos con otros obtenidos en procesos de investigación, ellos construyen mejores explicaciones y contribuyen más eficientemente a solucionar el problema.

Por otra parte, la evaluación del nivel de oposición a que llegaron muestran que más del 80\% del grupo experimental utilizó la categoría reserva y realizó contra-argumentaciones en el foro grupal a diferencia del grupo control cuyo porcentaje de utilización de esta categoría fue mucho menor. La inclusión dentro de la estrategia de una etapa para la contra-argumentación resultó positiva, porque necesariamente los estudiantes debían evaluar los argumentos de sus compañeros y al menos cuestionar con fundamento la posición de sus compañeros. Sin embargo, también hubo casos en que los estudiantes apoyaron la posición basados en la evidencia, lo cual también resultó válido para el ejercicio.

Además, la estrategia incluyó una etapa de consenso científico, en la cual los estudiantes evaluaron los argumentos y los contraargumentos que se habían construido hasta ese momento, con el fin de encontrar puntos de acuerdo y a partir de aquí estructurar en conjunto una ponencia argumentativa donde igualmente se consideraron las posibles excepciones a las hipótesis que defendieron, e incluso llegaron a recomendar la realización de experimentos más rigurosos para confirmar la hipótesis.

Es así que los resultados encontrados en esta investigación se pueden equiparar con los hallazgos del estudio realizado por Clark y Sampson (2008), quienes demostraron que aquellos estudiantes que durante un debate en línea realizaron contra-argumentos y réplicas, eran los que no solo tenían una mayor claridad del tema, sino que eran capaces de evaluar la calidad de los argumentos realizados por sus compañeros.

Sin embargo, si bien es cierto que el grupo experimental tuvo diferencias significativas en el nivel de oposición evaluado con respecto al grupo control, cabe resaltar que el $36.7 \%$ de los estudiantes del grupo experimental llegó al Nivel 3, solamente el 16.7\% llegó al Nivel 4 y ninguno al Nivel 5.
Estos resultados coinciden con los expuestos en investigaciones realizadas por Henao y Stipcich (2008) y Cardona y Tamayo (2009), quienes encontraron que los estudiantes al debatir sobre un problema científico tienen dificultades para presentar refutaciones coherentes, a pesar de haber realizado un entrenamiento previo. Además, para Tamayo (2011) la capacidad de oposición no solamente implica la claridad de los conocimientos, sino también flexibilidad intelectual y apertura a la crítica. De esta manera la oposición se considera como una forma más compleja de argumentación, puesto que no solamente se requiere un dominio conceptual, sino de un desarrollo del pensamiento crítico. De acuerdo a lo anterior, se puede afirmar que para alcanzar niveles de oposición más elevados, los estudiantes necesitan un entrenamiento más prolongado en actividades argumentativas.

Igualmente, el hecho de que el grupo control presente un nivel muy bajo en la capacidad de oposición demuestra que en entornos virtuales los estudiantes utilizan la refutación cuando es solicitada de forma abierta. Además, encontraron que el manejo y tipo de evidencia para defender una posición y la existencia de un compromiso para revisar diferentes puntos de vista, fueron resultados de foros de discusión diseñados ex profeso como espacios para el ensayo y desarrollo de habilidades argumentativas acompañados de la oportunidad brindada a los estudiantes de revisar la estructura de un buen argumento, previo a la participación en el foro.

\section{ConClusiones}

La novedad de esta investigación consistió en diseñar un ambiente virtual de aprendizaje basado en la argumentación dialógica para un curso de Biotecnología, en el que se consideró: (a) el guión o guía de actividades basado en un modelo argumentativo, (b) el contexto de aprendizaje situado y (c) los recursos tecno-didácticos que se fusionaron dentro del contexto virtual para facilitar la interacción y la interactividad didáctica. 
Igualmente, se propone una estrategia de aprendizaje basada en la argumentación dialógica en ambientes virtuales de aprendizaje que incluye cuatro etapas: (a) fundamentación científica, (b) desarrollo de la ponencia argumentativa, (c) contra-argumentación y (d) elaboración del consenso científico.

En el estudio realizado se pudo comprobar que tanto el diseño instruccional como la estrategia de aprendizaje basados en la argumentación dialógica planteados especialmente para este estudio resultaron más efectivos para mejorar las competencias argumentativas en cuanto a la estructura del texto, la calidad argumental y la capacidad para evaluar argumentos, que para aquellos estudiantes que participaron en el foro con una estrategia basada en aprendizaje colaborativo, la cual se venía implementando tradicionalmente en el curso virtual de Biotecnología.

En general, los resultados de esta investigación sugieren que la instrucción previa en la argumentación (enseñanza explícita de la argumentación, ejemplo de secuencia de un texto argumental, guía de interacción en el foro, ejercicio práctico de argumentación e instrucciones para participar en un foro argumentativo en línea) constituyó un factor positivo para alcanzar mayores niveles de conceptualización en la construcción de los argumentos, los cuales se basaron en la evidencia, se relacionaron los datos con la teoría y por lo tanto pudieron explicar objetiva y racionalmente el problema planteado. El uso de modelos argumentativos en la educación científica permitió a los estudiantes mejorar sus habilidades cognitivas y lingüísticas, lo que se evidenció en una mejor comprensión de la función de los argumentos y de la evaluación de la evidencia en la ciencia. Igualmente, mejoró sus habilidades de comunicación y de escritura, puesto que al tener que aplicar el modelo debían leer las intervenciones de sus compañeros, evaluarlas con otras fuentes y por tanto los obligó a tener más conciencia a la hora de escribir sus propias contribuciones.

Sin embargo, los resultados también indican que aunque los estudiantes del grupo experimental obtuvieron un mejor desempeño en el nivel de oposición que refleja el desarro- llo del pensamiento crítico, también indica que alcanzar niveles de oposición más elevados requiere una mayor dedicación en el entrenamiento de actividades de este tipo, dado que requiere no solamente tener un alto conocimiento del tema de discusión, sino también tener la capacidad de valorar con razones de juicio las intervenciones realizadas por otros estudiantes, lo que seguramente sucede cuando se alcanza un nivel de experto en la competencia argumentativa.

El estudio demuestra que aunque no es fácil dirigir un foro colaborativo en línea a partir de procesos estructurados de argumentación, la enseñanza de la argumentación ofrece algunas contribuciones potenciales como la externalización de los procesos cognitivos, el desarrollo del pensamiento crítico y soportes al desarrollo de criterios epistémicos, necesarios para la educación científica.

Se recomienda profundizar la investigación en los siguientes aspectos: (a) establecer si la retroalimentación de tipo correctivo y explicativo por parte de profesores y estudiantes tienen un efecto en la calidad de la argumentación y el nivel de oposición que los estudiantes adquieren en un ambiente virtual colaborativo, (b) realizar estudios longitudinales que permitan observar si los estudiantes que adquieren experticia en los debates en línea alcanzan niveles de oposición superiores y (c) indagar otras alternativas tecnológicas como softwares especializados que contribuyan al desarrollo del pensamiento lógico e insertarlas dentro de plataformas virtuales de aprendizaje.

Igualmente sería de interés indagar la relación entre la enseñanza de la investigación en línea con el desarrollo de competencias argumentativas. Aunque a nivel presencial se han encontrado relaciones positivas, no hay estudios realizados en ambientes virtuales de aprendizaje. Otro estudio que podría derivarse de esta investigación es establecer la relación entre la calidad de las interacciones alcanzadas en el grupo y el desarrollo del pensamiento crítico fundamental en la educación superior.

Esta investigación puede replicarse con estudiantes de modalidad a distancia y presen- 
cial, con el fin de profundizar sobre las implicaciones de la enseñanza de procesos argumentativos en el aprendizaje de conceptos y el desarrollo de habilidades científicas, lo que permitirá confirmar y ampliar los resultados.

\section{FIGURA 1}

ESQUEMA DEL DISEÑO INSTRUCCIONAL APLICADO A LOS GRUPOS EXPERIMENTAL Y CONTROL

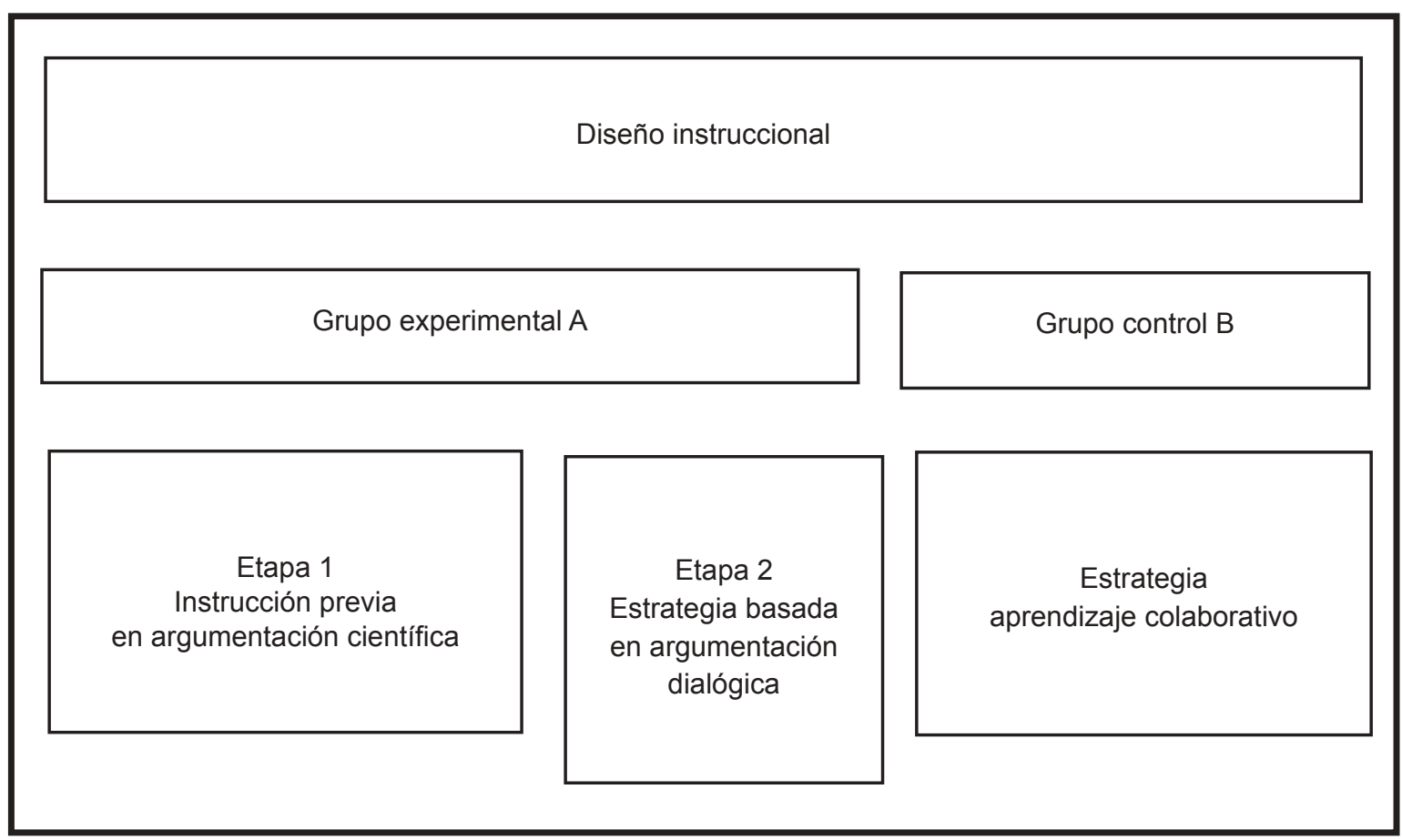




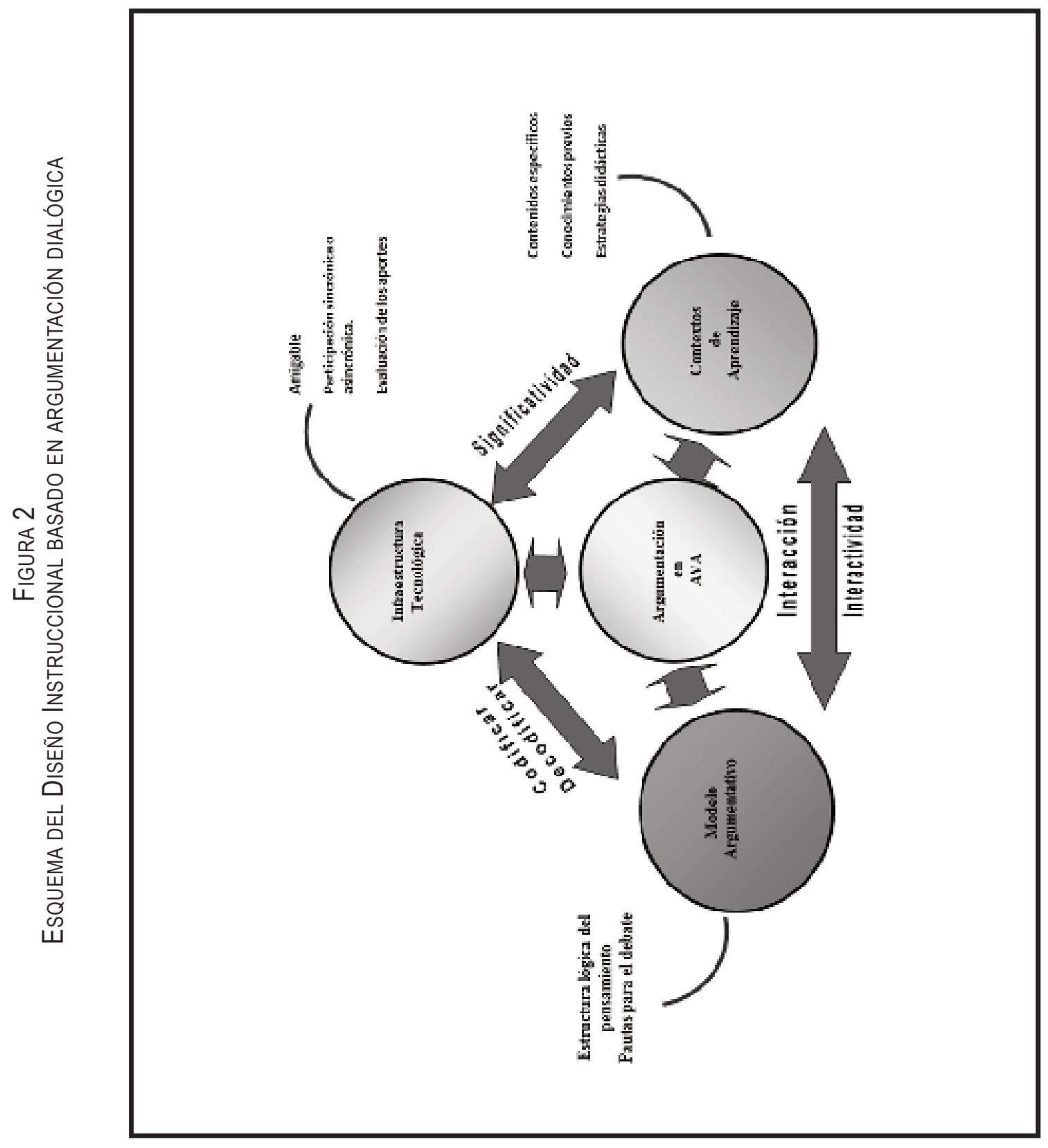




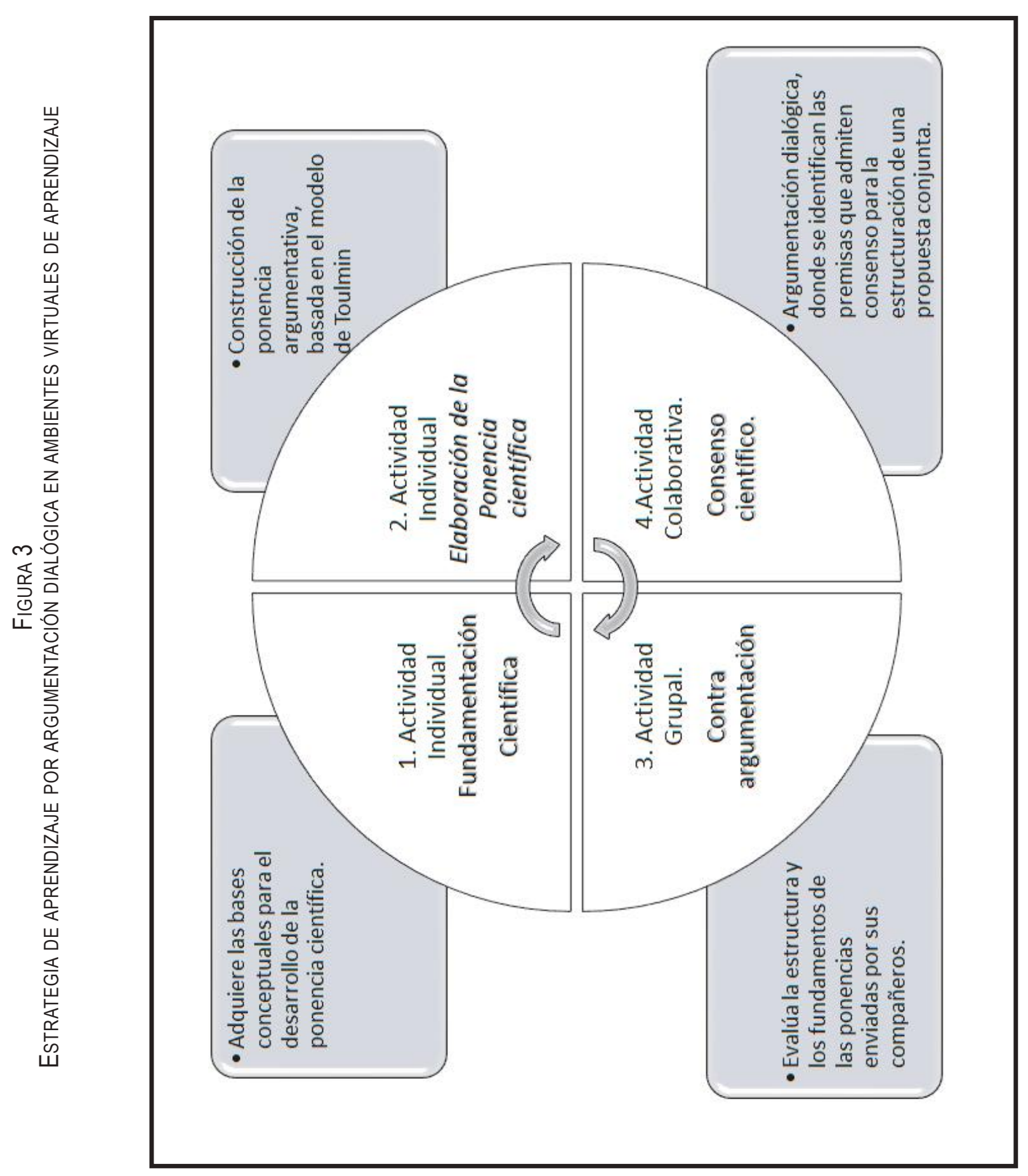


GRÁFICO 1

ESTRATEGIA DE APRENDIZAJE POR ARGUMENTACIÓN DIALÓGICA EN AMBIENTES VIRTUALES DE APRENDIZAJE

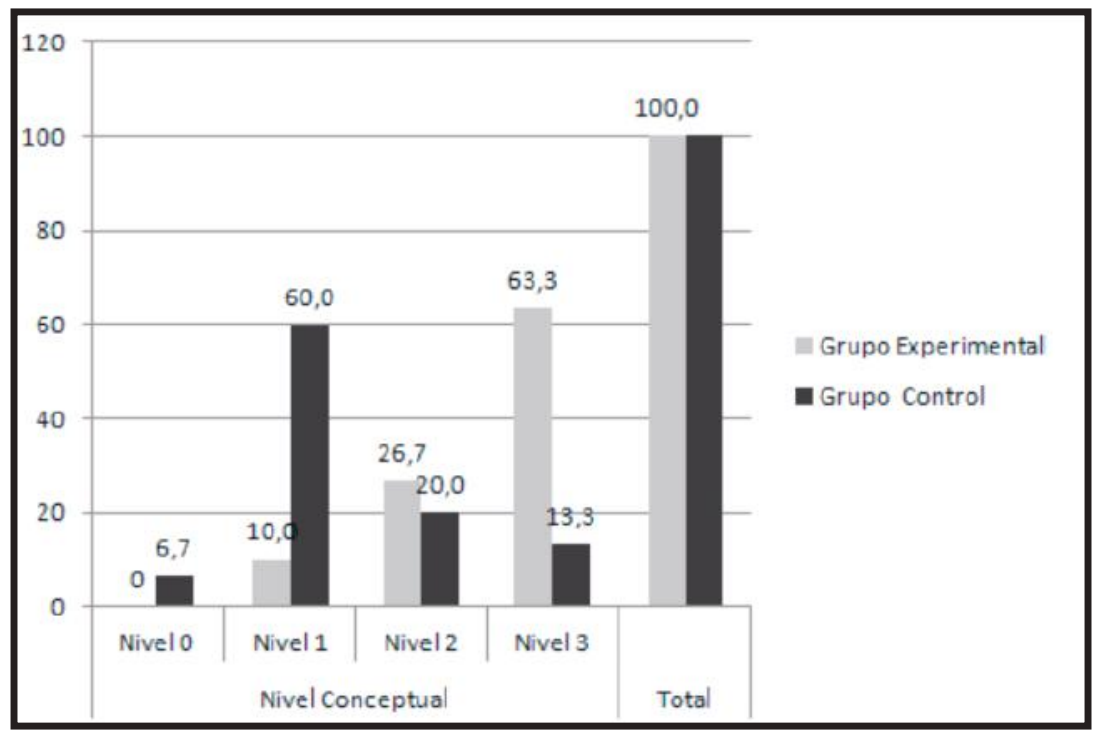

GRÁFICO 2

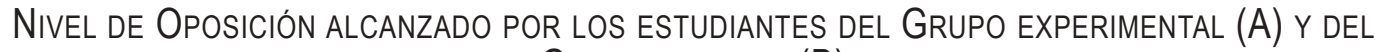

GRUPO CONTROL (B)

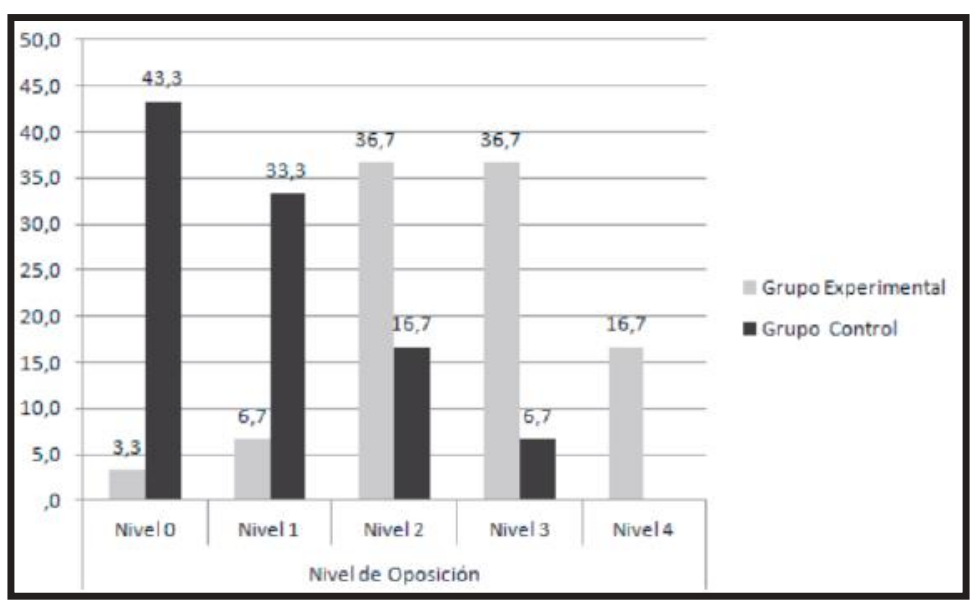




\section{REFERENCIAS BIBLIOGRÁFICAS}

Andriessen. J., Baker, M. \& Suthers, D. (2003). Argumentation, computer support, and the educational context of confronting cognitions. En J. Andriessen, M.J. Baker \& D. Suthers (Eds.), Arguing to learn: Confronting cognitions in computer-supported collaborative learning environments (pp. 1-25). Dordrecht, Netherlands: Kluwer Academic Publishers. Recuperado el 4 de julio de 2014 de http://ses-perso.telecomparistech.fr/baker/publications/ArticlesBakerP DF/2003/2003EtAl-a.pdf. http://dx.doi.org/10. 1007/978-94-017-0781-7_1.

Belland, B.R. (2010). Portraits of middle school students constructing evidence-based arguments during problem-based learning: the impact of computer-based scaffolds. Educational Technology Research and Development, 58(3), 285-309. http://dx.doi.org/10.1007/s11423-00 9-9139-4

Bouyias, Y.N. \& Starvros, D. (2012). Peer-monitoring vs. micro-script fading for enhancing knowledge acquisition when learning in computer-supported argumentation environments. Journal Computers \& Education, (59), 236249. http://dx.doi.org/10.1016/j.compedu.201 2.01 .001

Cardona, D. \& Tamayo, O. (2009). Modelos de argumentación en ciencias: Una aplicación a la genética [Models of argumentation in science: An application to genetics]. Revista Latinoamericana de Ciencias Sociales, Niñez y Juventud, 7(2), 1545-1571.

Campbell, D. \& Stanley, J. (1995). Diseños experimentales y cuasi experimentales en la investigación social [Experimental and quasiexperimental designs in social research] (7a. ed.). Buenos Aires, Argentina: Amorrortu Editores.

Clark, D. \& Sampson, V. (2008). Assessing dialogic argumentation in online environments to relate structure, grounds, and conceptual quality. Journal of Research in Science Teaching, 45(3), 283-321. http://dx.doi.org/10.100 2/tea. 20216

Dionne, C., Taasoobshirazi, G., Hendricks, S. \& Hickey, D. (2008). Argumentation: A strategy for improving achievement and revealing scientific identities. International Journal of Science Education, 30(6), 837-861. http://dx. doi.org/10.1080/09500690701411567

Dole, J. \& Sinatra, G. (1998). Reconceptualizing change in the cognitive construction of knowledge. Educational Psychologist, 33(2/3), 109128. http://dx.doi.org/10.1207/s15326985ep33 $02 \& 3 \_5$

Driver, R., Newton, P. \& Osborne, J. (2000). Establishing the norms of scientific argumentation in classrooms. Science Education, 84, 287-313. http:// dx.doi.org/10.1002/(SICI)1 098-237X(200005)84:3<287::AID-SCE 1>3. 0.CO;2-A

Duschl, R., Ellenbogen, K. \& Erduran, S. (1999, Abril). Understanding dialogic argumentation. Paper presented at the Annual Meeting of American Educational Research Association. Montreal.

Erduran, S., Ardac, D. \& Yakmaci-Guzel, B. (2006). Learning to teach argumentation: Case studies of pre-service secondary science teachers. Eurasia Journal of Mathematics, Science and Technology Education, 2(2), 1-14.

Gunawardena, C. \& Lowe, M., Anderson, T. (1997). Analysis of a global online debate and the development to fan interaction analysis model for examining social construction of knowledge in computer conferencing. Journal of Educational Computing Research, 17, 397431 .

Henao, B.L. \& Stipcich, M.S. (2008). Educación en ciencias y argumentación: La perspectiva de Toulmin como posible respuesta a las demandas y desafíos contemporáneos para la enseñanza de las Ciencias Experimentales [Education in sciences and argumentation: The per- 
spective of Toulmin as a possible answer to the demands and contemporary challenges to the teaching of experimental sciences]. Revista Electrónica de Enseñanza de las Ciencias, 1(7).

Hogan, K., Nastasi, B.K. \& Pressley, M. (1999). Discourse patterns and collaborative scientific reasoning in peer and teacher-guided discussions. Cognition \& Instruction, 17(4), 379432. http:// dx.doi.org/10.1207/S1532690XCI 1704_2

Jiménez, M.P., Bugallo, A. \& Duschl, R.A. (2000). «Doing the lesson» or «doing science»: Argument in High School Genetics', Science Education, 84(6), 757-792. http://dx.doi.org/ 10.1002/1098-237X(200011)84:6<75 7::AIDSCE5 $>3$.0.CO;2-F

Jiménez-Aleixandre, M.P. (2005). La construcción del discurso científico socialmente contextualizado [The construction of the socially contextualized scientific discourse]. Revista Enseñanza de las Ciencias, Número especial. Recuperado el 12 de marzo de 2015 de http://dd d.uab.cat/pub/edlc/edlc_a2005nEXTRA/edlc_ a2005nEXTRAp511condis.pdf.

Jeong, D. (2011). Sequential analysis of scientific argumentation in asynchronous online discussion environments. Analyzing interactions in CSCL. Computer Supported Collaborative. Learning Series, 12(2), 207-233. http://dx. doi.org/10.1007/978-1-4419-7710-6_10

Kinsley, D. \& Firuz, H (2011). Students' decision steps in meta-cognitive learning in free online groups (metal-frog): a case study. The Turkish Online Journal of Distance Education. TOJDE, 12(1), 152-165.

Kollar, I., Fischer, G. \& Hesse, F.W. (2010). Collaboration scripts a conceptual analysis. Educational Psychology Review, 18(2), 159-185. http://dx.doi.org/10.1007/s10648-006-90 07-2

Kuhn, D. (2008). Beyond control of variables: What needs to develop to achieve skilled scientific thinking? Cognitive Development, 23
(4), 435-451. http://dx.doi.org/10.1016/j.co g dev.2008.09.006

Linn, M., Shear, M., Bell, P. \& Slotta, J. (1999). Organizing principles for science education partnerships: Case studies of students' learning about 'rats in space'and 'deformed frogs'. Educational Technology Research and Development, 47(2), 61-84. http://dx.doi.org/10.10 07/ BF02299466

Litao, S. (2000). El potencial de la argumentación en la construcción del conocimiento [The potential of the argumentation on the construction of knowledge]. Desarrollo Humano, 43, 332-360.

Marafioti, R. \& Santibáñez, C. (2008). Teoría de la argumentación: 50 años de Perelman y Toulmin [Argumentation theory: 50 years of Perelman and Toulmin]. Buenos Aires: Biblos.

Mercer, N. (2001). Palabras y mentes [Words and minds]. Barcelona: Paidós.

Osborne, J.S. (2004). Enhancing the quality of argumentation in science classrooms. Journal of Research in Science Teaching, 41, 9941020. http://dx. doi.org/10.1002/tea.20035

Peñaloza, E., García, C. \& Espinoza, M. (2011). La argumentación como recurso para el aprendizaje colaborativo en internet: Una propuesta [The argumentation as a resource for collaborative learning on the internet: A proposal]. Universidad Autónoma Metropolitana. Cuajimalpa.

Sánchez, A., Sánchez, L., Méndez, J. \& Puerta, C. (2013). Alfabetización académico-investigativa: Citar, argumentar y leer en la red [Academic-investigative literacy: Quote, argue and read online]. Revista Lasallista de Investigación, 10(2), 151-163. http://dx.doi.org/10.695 29816015

Schwarz, B. \& Perret, C. (2006). Escalate: The white book. Jerusalén. Israel: The Hebrew University of Israel.

Simon, S., Erduran, S. \& Osborne, J. (2006). Learning to teach argumentation. Research and 
development in the science classroom. International Journal of Science Education, 28, 235-260. http://dx.doi.org/10.108 0/09500690 500336957

Skoumios, M. (2008). Sociocognitive conflict processes in science learning: benefits and limits. Journal of Baltic Science Education, 7(3), 165174.

Tamayo, O.E. (2011). La argumentación como constituyente del pensamiento crítico en niños [The argumentation as a constituent of critical thinking in children]. Revista Hallazgos, 9(17),
211-233. http://dx.doi.org/10.15332/s1794-38 41.2012.0017.10

Toulmin, S. (1958). The uses of argument..Cambridge, England: Cambridge University Press.

Toulmin, S., Rieke, R. \& Janik, A. (1984). An introduction to reasoning. New York-London: Collier Macmillan Publishers.

Wen, H., Wei, Ch. \& Shing, N. (2012). The effectiveness of using procedural scaffoldings in a paper-plus-Smartphone collaborative learning context. Journal Computers \& Education, 59 (2), 250-259. http://dx.doi.org/10.1016/j.compedu.2012.01.015.

Universidad Nacional Abierta y a Distancia (UNAD)

Universidad de Nariño

Nariño - Colombia

Fecha de recepción: 11 de agosto de 2015

Fecha de aceptación: 9 de mayo de 2016 
